\title{
An unattended device for sleep-related breathing disorders: validation study in suspected obstructive sleep apnoea syndrome
}

\author{
M. Zucconi, L. Ferini-Strambi, V. Castronovo, A. Oldani, S. Smirne
}

\begin{abstract}
An unattended device for sleep-related breathing disorders: validation study in suspected obstructive sleep apnoea syndrome. M. Zucconi, L. Ferini-Strambi, V. Castronovo, A. Oldani, S. Smirne. COERS Journals Ltd 1996.

ABSTRACT: Portable devices for the diagnosis of obstructive sleep apnoea (OSA) are considered to be an acceptable alternative to polysomnography (PSG), but their validation is essential. The aim of our study was to validate a device specifically designed for OSA diagnosis.

Twenty nine suspected OSA patients were studied with simultaneous nocturnal PSG and an unattended recording device (MicroDigitrapper-S) (M-S). The device measured body position, snoring sound, oronasal flow, thoracic and abdominal effort, heart rate and percentage arterial oxygen saturation $\left(\mathrm{Sa}_{\mathrm{a}}, \mathrm{O}_{2} \%\right)$. We compared the apnoea plus hypopnoea index (AHI) and $\mathrm{Sa}_{\mathrm{a}} \mathrm{O}_{2} \%$ results of PSG with that of the system's automatic analysis (M-SA). We also performed a semiautomatic analysis (MSS) with visual editing of the raw data. Results at different AHI cut-off levels were analysed to obtain an indication of accuracy in diagnosis and severity.

Both M-SA and M-SS showed a sensitivity and specificity of $100 \%$ at the cut-off level of AHI $>10$. When increasing the cut-off levels, M-SA sensitivity decreased (55\% for $\mathrm{AHI}>40)$, while specificity remained high $(\mathbf{9 5 \%})$. This was improved to a clinically acceptable level of agreement by M-SS analysis (sensitivity $91 \%$ and specificity $94 \%$ ).

In conclusion, the MicroDigitrapper-S device showed a good sensitivity and specificity for the diagnosis of OSA. However, the device could not predict the severity of OSA precisely enough. In severe cases (apnoea plus hypopnoea index $>40$ ), semiautomatic scoring was necessary to obtain a more accurate detection of the severity of the disease.

Eur Respir J., 1996, 9, 1251-1256.
\end{abstract}

Obstructive sleep apnoea syndrome (OSA) is the most common type of sleep-disordered breathing $[1,2]$. Until now, nocturnal polysomnography (PSG) has been considered the gold standard for the diagnosis and estimation of the severity of the syndrome [2]. However, standard PSG is a time-consuming diagnostic procedure with high costs that is essentially limited to Sleep Disorders Centres. This often results in long waiting periods and, consequently, delayed treatment for OSA patients.

Previous reports [3-5] have discussed the necessity for an easy and widespread access to OSA diagnosis in terms of procedures and devices that have to be less expensive and simpler, but with an acceptable positive and negative predictive power with respect to PSG. Unattended and portable devices for recording respiratory and sleep parameters have recently come onto the market; however, validation studies, demonstrating their accuracy in terms of sensitivity and specificity compared to PSG, are still limited [3]. In fact, a recent literature review and indications of the American Sleep Disorders Association for standard of practice, reported that portable recording may be acceptable for the assessment of OSA only if the equipment is validated and for particular situations $[4,5]$.
Sleep Disorders Center, Dept of Neurology, State University and IRCCS H San Raffaele, Milan, Italy.

Correspondence: M. Zucconi

Sleep Disorders Center

Dept of Neurology

San Raffaele Hospital

Via Prinetti 29

20127 Milan

Italy

Keywords: Obstructive sleep apnoea unattended device

validation study

Received: August 101995

Accepted after revision March 101996
Thus, we planned a validation study of a relatively new device for OSA, the MicroDigitrapper-S (M-S) (Synectics Medical, Stockholm), using a configuration intended to measure primarily respiratory parameters. This represents the first step in the evaluation of a device that may be used on patients at home.

\section{Materials and methods}

\section{Patients}

We studied 30 consecutive patients, referred to the Milan San Raffaele Hospital Sleep Disorders Center, for habitual snoring and suspected OSA, based on symptoms of daytime sleepiness and/or witnessed snoring and periods of cessation of breathing during sleep. All candidates underwent a nocturnal evaluation of sleep and breathing using a traditional full nocturnal PSG and, simultaneously, the MicroDigitrapper-S device. Moreover, each patient completed the Epworth Sleepiness Scale (ESS) to assess and quantify daytime sleepiness [6]. 


\section{Polysomnography}

A standard examination was performed using two electroencephalogram (EEG) channels (C3-A2 and 02-A1), two electro-oculograms (EOG, left and right) connected with a reference (A1 or A2), one submental electromyogram (EMG), one electrocardiogram (ECG) lead for heart rate, and one intercostal EMG for respiratory effort (generally the 6th intercostal space muscle). Respiration was monitored by recording thoracic and abdominal effort using piezoelectric sensors and oronasal flow by means of a thermistor. Snoring sounds were recorded using a microphone connected to an amplifier, and body position was checked by a video-recording. Percentage arterial oxygen saturation $\left(\mathrm{Sa}_{\mathrm{a}} \mathrm{O}_{2} \%\right)$ was detected using a finger probe oximeter (Model 3700, Ohmeda, Denver, CO, USA). PSG paper speed was set at $10 \mathrm{~mm} \cdot \mathrm{s}^{-1}$ to allow correct sleep staging.

\section{The MicroDigitrapper-S (M-S)}

The M-S is a microprocessor-based data logging device that measures as many as eight different physiological parameters simultaneously for an entire night (size 150× $28 \times 129 \mathrm{~mm}$, weight $560 \mathrm{~g}$ ). Its major in-built components are a microcomputer, 1-4 Mbytes Solid State memory, the screen, the oximeter and amplifiers for analogue signals. The data are continuously stored as 8 bit samples on solid state memory (1-4 Mb) and no data compression techniques are employed. The raw data are not processed during the recording and the device does not manipulate the data in any way. Sampling rate can be set for each channel and it is dependent on the parameter recorded (i.e. $8 \mathrm{~Hz}$ for respiration, $1 \mathrm{~Hz}$ for body position, $4 \mathrm{~Hz}$ for snoring, and $2 \mathrm{~Hz}$ for pulse and $\mathrm{Sa}, \mathrm{O}_{2} \%$ ). A computer is not required to set up the recording; automatic calibration and verification of signals is performed directly on the recorder. The device is modular; there is no set configuration except for pulse rate and oxygen saturation from the in-built oximeter (MiniOx V; Mini Safety Appliance, MSA Inc.).

For this study, the following configuration was used: body position (a 5-way encapsulated mercury switch); snoring sound (a surface encased skin-throat microphone); oronasal flow (thermistor); thoracic and abdominal respiration (piezoelectric sensors); pulse rate; $\mathrm{Sa}_{2} \mathrm{O}_{2} \%$ (finger probe oximeter). For detecting airflow, two similar thermistors as well as two sets of respiration belts (piezoelectric) were used both for PSG and M-S.

The recorded data were retrieved and transferred to a standard personal computer (PC) where the review, analysis and editing were performed using the Multigram SA (TM, Synectics Soft., Stockholm \& Dallas) software package.

All the analyses are configurable using the system constant menu that allows the users to change the different parameters that are then used for the automatic analysis (i.e. sensitivities and thresholds). In this study, the same default settings and constants were used for all patients.

Respiration analysis can be performed on any and/or on all respiration channels. Respiratory events were marked if a decrease of the signal amplitude of at least
$40 \%$ with a minimum length of $10 \mathrm{~s}$ was found. Since most of the respiratory events were obstructive in type, the analysis was performed on the airflow signal, without differentiating central or mixed events from the obstructive ones. For oxygen saturation data, the $4 \%$ dip analysis was chosen, where, all dips of at least $4 \%$ with a length of $\geq 2 \mathrm{~s}$, were counted as valid desaturations.

\section{Scoring procedure}

Three different types of analysis were performed: PSG scoring, M-SA scoring and M-SS scoring.

$P S G$ scoring. All the recordings were hand-scored by $30 \mathrm{~s}$ epochs analysis following international criteria [7] for sleep stages assessment and standard parameters for breathing analysis based on respiratory effort, airflow and oximetry findings [8]. Apnoea was defined as a complete cessation of airflow for at least $10 \mathrm{~s}$ and hypopnoea as a decrease in oronasal flow greater than $40 \%$ and lasting for at least $10 \mathrm{~s}$. Since there is little clinical reason to separate apnoea from hypopnoea, we grouped them together. These definitions do not include criteria of oxygen desaturation episodes greater than $4 \%$, which were considered by visual analysis of a separate plotted paper for $\mathrm{Sa}, \mathrm{O}_{2} \%$ with a speed of $10 \mathrm{~cm} \cdot \mathrm{h}^{-1}$.

The M-S tested did not include EEG monitoring and, therefore, we were unable to calculate the total sleep time (TST). We thus defined the apnoea plus hypopnoea index (AHI) as the total number of events per total time in bed (in hours), calculated from light-off to lighton. We also calculated the oxygen desaturation index (ODI) based on the total time in bed. The total number of apnoea+hypopnoea (respiratory events (RE)), the total number of oxygen desaturation dips (desaturation events (DE)), the mean level of $\mathrm{Sa}, \mathrm{O}_{2} \%$ dips (mean low $\mathrm{Sa}_{2} \mathrm{O}_{2} \%$ ) and the mean of the minimum $\mathrm{Sa}_{\mathrm{a}} \mathrm{O}_{2} \%$ dips (mean min $\mathrm{Sa}, \mathrm{O}_{2} \%$ ) were also calculated for each patient.

$M-S A$ scoring. The same variables as PSG were calculated by the automatic analysis according to the criteria described. Snoring sound, heart rate and body position were also automatically analysed but not used for this study. They are equally important variables, especially for the visual analysis.

$M$-SS scoring. A visual check was performed on the PC screen of the automatic analysis, where the reviewer is allowed to add, delete or change any respiratory event. On the basis of previous works involving completely automatic analysis without the evaluation of manual (visual) over-reading of the raw data $[9,10]$, it was decided to review each recording, in a blind manner, in order to check possible over- or underestimation of respiratory events. For this procedure, the automatic analysis serves as a base for scored apnoea and hypopnoea events; the reviewer, having an overview of all channels recorded, i.e. snoring sound more elevated at the end of events, pulse rate and $\mathrm{Sa}_{2} \mathrm{O}_{2} \%$ synchronous variations with respiratory events (fig. 1), checks these choices, taking into account possible artifacts and errors. This procedure of reviewing and correcting the previous automatic analysis of respiratory events takes approximately 30-45 min. 


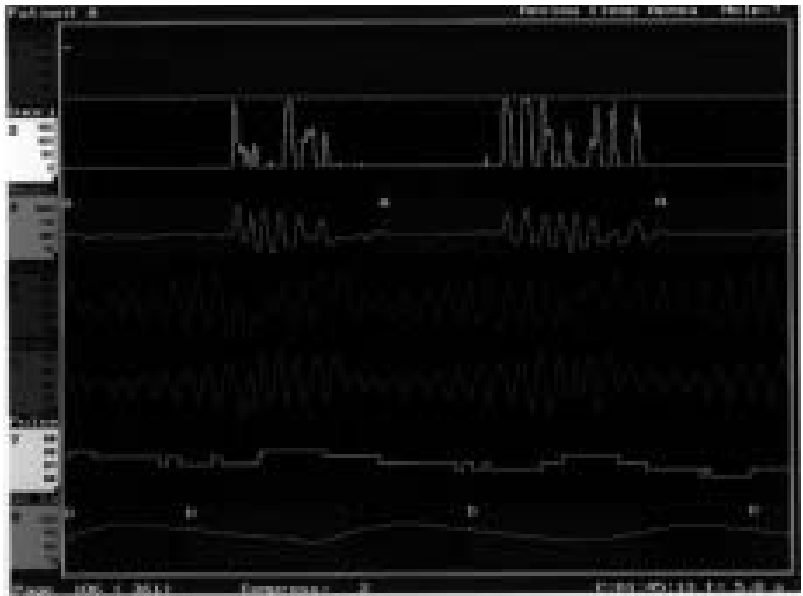

Fig. 1. - Overview of raw data of all channels recorded. 1: body position; 2: snoring sound; 4: oro-nasal respiration; 5 : thoracic respiration; 6: abdominal respiration; 7: pulse rate; 8 : arterial oxygen saturation $\left(\mathrm{Sa}_{\mathrm{a}} \mathrm{O}_{2}\right)$. Numbers appearing in the figure from channel 1 to 6 are arbitrary units.

The software of the M-S again calculates the data considering the changes and the new results are then printedout. In our study, the PSG analysis and the M-SS scoring were performed by two independent scorers, blind to the results of the other procedure at the time of data analysis. The interobserver variability of the PSG respiratory events scoring was very low (less than 5\%) and nonsignificant. Moreover, as a control of the semiautomatic analysis, the data were reanalysed by a third scorer, blind to the previous results, who showed an interscorer agreement of $95 \%$.

\section{Statistical analysis}

Due to the fact that the data were not normally distributed, all the variables were compared using the Wilcoxon's signed-rank test. The significance of the results was adjusted applying the Bonferroni correction for comparison of the three groups of data, and a $\mathrm{p}<0.016$ was considered significant. Differences between the measures (PSG - M-SA and PSG - M-SS) were also calculated with the $95 \%$ confidence interval $(95 \% \mathrm{CI})$ and the plots of differences between the methods of analysis of AHI against their mean were given according to the method of Bland and Altman [11]. The sensitivity, specificity, positive predictive value (PPV) and negative predictive value (NPV) of M-SA and M-SS results were calculated for different cut-off values of AHI using a contingency analysis. The lack of well-defined cut-offs for AHI and ODI makes the assessment of validity of the device as difficult as the PSG interpretation. Nevertheless, this is of low clinical importance. But it is possible that small differences in AHI between the systems, which are clinically unimportant, may influence the sensitivity and specificity of the device. To avoid this, a test of diagnostic agreement was applied as previously used by WhITE et al. [12]. This test considers the differences in scoring to be substantial only when at least \pm 10 events $\cdot h^{-1}$ are found between the two methods (for values of $\mathrm{AHI}<40$ ). For AHI $\geq 40$ on both systems, it was considered that there was diagnostic agreement.
Table 1. - Patient characteristics (according to PSG data)

\begin{tabular}{lrl}
\hline Age yrs & $53 \pm 12$ & $(23-68)$ \\
Sex M/F & \multicolumn{2}{c}{$20 / 9$} \\
BMI $\mathrm{kg} \cdot \mathrm{m}^{-2}$ & $30.7 \pm 6.2$ & $(18.3-44.2)$ \\
Snoring history yrs & $15 \pm 11$ & $(1-43)$ \\
ESS score & $12 \pm 3$ & $(5-20)$ \\
Patients with n $(\%)$ & 9 & $(31)$ \\
AHI $<5$ & 19 & $(65)$ \\
AHI $>10$ & 16 & $(55)$ \\
AHI $>20$ & 11 & $(38)$ \\
AHI $>40$ & & \\
\hline
\end{tabular}

Values are presented as mean $\pm \mathrm{SD}$, and range in parenthesis. PSG: polysomnography; M: male; F: female; BMI: body mass index; AHI: apnoea plus hypopnoea index (events.h ${ }^{-1}$ of total time in bed); ESS: Epworth Sleepiness Scale (score range 0-20) [6].

\section{Results}

From the original 30 patients, the data of one patient was not considered in the study because only $4 \mathrm{~h}$ of acceptable airflow signal was obtained from the M-S. Anthropometric and respiratory (PSG) data of the 29 patients (20 males and 9 females) are summarized in table 1 . Sixteen of the 29 patients showed daytime sleepiness according to the ESS: they all had a score greater than 11 , and 9 of them (with an AHI >40) had a score $\geq 15$ (a value that indicates substantial daytime somnolence) [6]. Most of the patients were overweight and with a prolonged history of habitual snoring. Nine of them ( 5 males and 4 females) showed no significant respiratory events (AHI $\leq 5)$. Nineteen subjects had an AHI >10, equally distributed among males and females; 11 patients showed a severe OSA (AHI >40) and five of them also had an important night-time hypoxia (mean level of $\mathrm{Sa}_{\mathrm{a}} \mathrm{O}_{2}$ dips $<80 \%$ ).

Mean AHI values were significantly different when comparing PSG and M-SA; a lower mean resulted for M-SA (table 2). No significant differences were found when comparing PSG and M-SS. The confidence interval band with M-SS was narrower $( \pm 11)$ and the mean of the differences was lower with respect to M-SA. The differences plot showed progressively less agreement for M-SA with respect to PSG with increasing AHI (fig. 2), whilst M-SS data demonstrated an acceptable agreement for almost all values (included between 95\% CI) (fig. 3). Moreover, there was a significant difference in the total number of RE between PSG and M-SA, but not between PSG and M-SS. There was no significant difference between PSG and M-SA for ODI, total number of DE, mean low $\mathrm{Sa}_{2} \mathrm{O}_{2} \%$, and mean min $\mathrm{Sa}_{2} \mathrm{O}_{2} \%$ (table 2).

The data presented in table 3 show that for M-SA the sensitivity was high for cut-off values of $10(100 \%)$ or $20(94 \%)$ but decreased (as low as 54\%) when increasing the cut-off to 40 . The specificity ranged 100-95\% for all the AHI levels used as cut-off. When the M-SS scoring was considered (table 3), an important increase in sensitivity was obtained, maintaining a high specificity for all values of AHI. Thus, M-SS improved the completely automatic scoring, especially for the evaluation of severe OSA.

The PPV and the NPV obtained with the M-SA at different cut-off levels are shown in table 3. Good PPV 
Table 2. - Polysomnographic (PSG), automatic (M-SA) and semiautomatic (M-SS) scoring of the portable device and mean difference between measures

\begin{tabular}{|c|c|c|c|c|c|}
\hline & PSG & M-SA & M-SS & PSG minus $\mathrm{MS}-\mathrm{A}$ & PSG minus MS-S \\
\hline AHI & $31.5 \pm 26.1$ & $25.2 \pm 19.4^{* *}$ & $31.8 \pm 27.5$ & 6.27 (26.3 to -13.8$)$ & 0.08 (11.5 to -11.6$)$ \\
\hline ODI & $30.2 \pm 25.4$ & $29.4 \pm 25.8$ & - & 0.81 (21.1 to -19.4$)$ & \\
\hline $\mathrm{RE} \mathrm{n}$ & $228.8 \pm 191.8$ & $182.3 \pm 140.0 *$ & $227.9 \pm 198.2$ & $46.4 \quad(280$ to -189$)$ & $(86$ to -84$)$ \\
\hline $\mathrm{DE} n$ & $220.1 \pm 189.1$ & $203.3 \pm 178.2$ & - & $(156$ to -122$)$ & \\
\hline Mean low $\mathrm{Sa}_{\mathrm{a}, \mathrm{O}_{2}} \%$ & $86.0 \pm 8.1$ & $85.6 \pm 5.7$ & - & 0.41 (13.3 to -12.5$)$ & \\
\hline Mean $\min \mathrm{Sa}, \mathrm{O}_{2} \quad \%$ & $73.1 \pm 16.4$ & $73.2 \pm 14.3$ & - & $-0.07 \quad(6.9$ to -7.0$)$ & \\
\hline
\end{tabular}

Values are presented as mean \pm SD, and $95 \%$ confidence interval in parenthesis. M-S: MicroDigitrapper-S; AHI: apnoea plus hypopnoea index (events $\cdot h^{-1}$ of total time in bed); ODI: oxygen desaturation index $\left(\mathrm{O}_{2}\right.$ desaturation dips $\cdot \mathrm{h}^{-1}$ of total time in bed); RE: respiratory events; DE: desaturation events; Mean low $\mathrm{Sa}_{\mathrm{a}} \mathrm{O}_{2}$ : the mean of low dips of $\mathrm{Sa}_{\mathrm{a}} \mathrm{O}_{2} \%$; Mean min $\mathrm{Sa}_{\mathrm{a}} \mathrm{O}_{2}$ : mean of the minimum values of $\mathrm{Sa}_{\mathrm{a}} \mathrm{O}_{2} \%$. Significance was tested by Wilcoxon's signed-rank test with Bonferroni adjustment when appropriate $(*: \mathrm{p}<0.01 ; * *: \mathrm{p}<0.009)$.

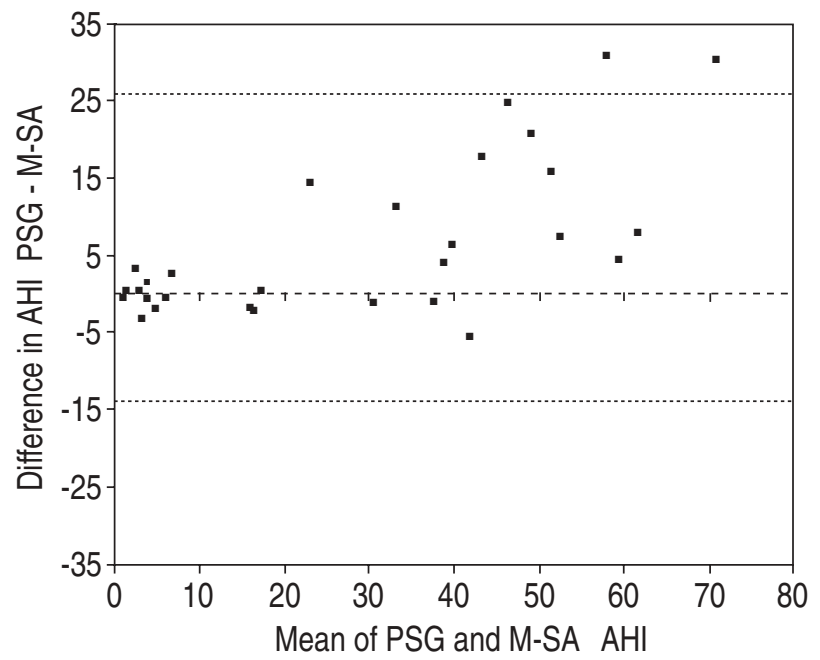

Fig. 2. - Difference between the two analyses (PSG - M-SA) to the mean of the two scores for AHI. The upper and lower $95 \%$ confidence interval $(95 \% \mathrm{CI})$ of the difference are indicated by horizontal lines. AHI: apnoea plus hypopnoea index (events $\cdot \mathrm{h}^{-1}$ of total time in bed); PSG: polysomnography; M-SA: automatic scoring with MicroDigitrapper-S.

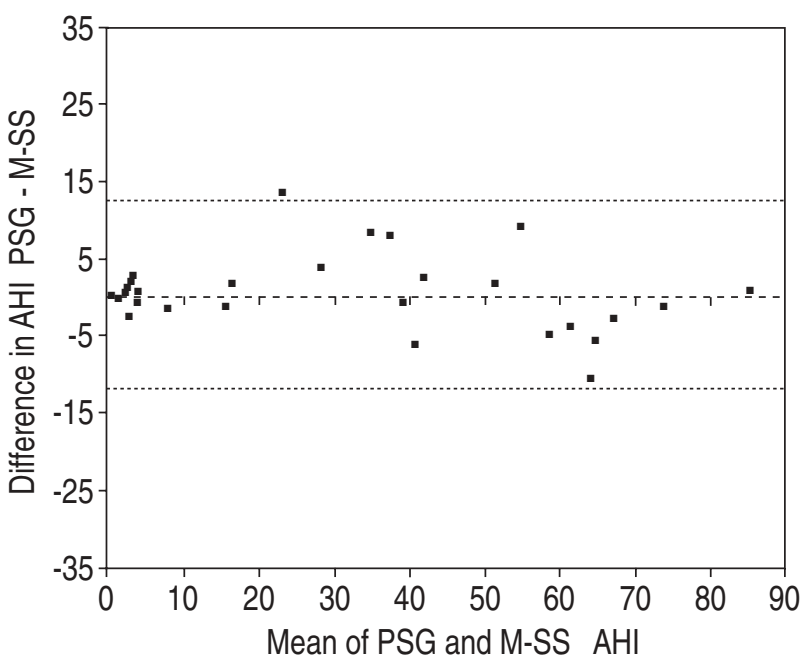

Fig. 3. - Difference between the two analyses (PSG - M-SS) to the mean of the two scores for AHI. The upper and lower $95 \%$ confidence interval $(95 \% \mathrm{CI})$ of the difference are indicated by horizontal lines. M-SS: semiautomatic scoring with MicroDigitrapper-S. For further definitions see legend to figure 2 . and NPV were obtained at low cut-offs, but at AHI $>40$ PPV was $86 \%$ and NPV 94\%. M-SS increased PPV to $91 \%$ but decreased NPV to $77 \%$. Because of the difference between PSG and M-SA results for patients with a severe OSA, the data of this group $(n=11)$ were re-examined. Six patients showed substantially different values for AHI due to the underscoring of M-SA which M-SS corrected. Reviewing the PSG and clinical data of these six patients, it was found that they were the most severe cases (AHI >50), obese and with an overlap syndrome (associated restrictive pulmonary defect or chronic obstructive disease) [13]. For these patients, there were several events of borderline duration (8-10 s) or with only a decrease in oronasal flow amplitude, less than $40 \%$, but with arousals and oxygen desaturations. In these cases, there was a tendency for the visual scorers, both for PSG and M-SS scoring, to mark borderline events as apnoea or hypopnoea, while M-SA did not. This finding was confirmed by the test of diagnostic agreement: with M-SA an underestimation was obtained for five patients and no overestimation, and with M-SS there was only one overestimation.

\section{Discussion}

Our results indicate that the M-S unattended monitor for suspected OSA patients has a high sensitivity and specificity when used for diagnostic and screening purposes, i.e. to detect subjects with AHI greater than 10 or 20. Since the latter cut-off value is considered significant enough to establish treatment when clinically indicated, and it has been shown to be relevant in mortality prognosis [14], M-S appears to be useful for the diagnosis of OSA or habitual snoring without respiratory events in a sample of subjects referred to a Sleep Disorders Centre with suggestive symptoms of OSA. When the severity of the syndrome was considered, the discriminating power was not good, especially in terms of sensitivity. A positive M-S result was probably associated with a correct OSA diagnosis. However, as the cut-off level was raised, the number of false negatives increased. We concluded that, whilst satisfactory for the diagnosis, the automatic analysis could not predict the exact severity of the disorder in OSA patients. The discriminating power of the M-S was improved by visual analysis of the data. This scoring procedure permitted a simultaneous view of all respiratory parameters, similar 
Table 3. - Sensitivity, specificity and predictive values of the MicroDigitrapper-S (M-S)

\begin{tabular}{lcccccc}
\hline & AHI $>10$ & MS-A & & & MS-S \\
& AHI $>20$ & AHI $>40$ & AHI $>10$ & AHI $>20$ & AHI $>40$ \\
\hline Sensitivity $\%$ & 100 & 94 & 55 & 100 & 94 & 91 \\
Specificity $\%$ & 100 & 100 & 95 & 100 & 92 & 94 \\
PPV \% & 100 & 100 & 86 & 100 & 94 & 91 \\
NPV \% & 100 & 93 & 94 & 100 & 92 & 77 \\
\hline
\end{tabular}

MS-A: automatic scoring; MS-S: semiautomatic scoring; AHI: apnoea plus hypopnoea index (events.h-1 of total time in bed); PPV: positive predictive value; NPV: negative predictive value.

to polysomnography scoring. Moreover, utilizing this scoring-method, only one false positive and one false negative remained.

MicroDigitrapper-S showed a good patients compliance with a low failure rate of only $3.3 \%$ due to technical problems. The device is relatively simple to apply; the hook-up and the testing of efficient functioning of the sensors takes around $20-30 \mathrm{~min}$. It is a noninvasive procedure and of sturdy structure to allow unattended examinations in or out of the laboratory. From an economic point of view, the device has a relatively low price that permits, if associated with clinical data and visual analysis, not only screening but also diagnostic procedures. This may allow attended PSG to be saved for other more complex sleep breathing disorders or for testing therapeutic choices.

We examined recent studies of portable devices to get an idea of accuracy compared with other systems; M-S gave better results at lower AHI levels [12, 15-17]. Other devices had high sensitivity and specificity, but in these cases only low cut-offs were examined $[9,10,18$, 19]. Some studies have compared results of automatic and visual scoring [20-22] but these are limited. We consider it is very important that potential users should understand the necessity to inspect traces visually, and that validation studies should present automatic analysis results at all cut-off levels if available.

There are some points that should be discussed when we look at this validation study. The number of patients studied was not high but we considered it sufficient, since there was a wide range of OSA severity with male and female representation. We are also concerned about the relatively small number of OSA subjects with AHI between 10 and 40 (eight patients), which show the greatest potential for confusion in diagnosis. However, for these patients both M-SA and M-SS analyses demonstrated good accuracy in the diagnosis.

A further point is that the number of failures could increase in the home setting. However, in our in-laboratory study, the M-S recordings were not checked or adjusted throughout the night.

Furthermore, some studies have shown the proportion of failures among attended and unattended studies to be similar [18].

The choice of habitual snorers and suspected OSA patients as sample subjects, instead of the general population, could be considered a limitation of this study. Our data showed that M-S was accurate, especially with subjects with few respiratory events, indicating a $100 \%$ sensitivity and specificity for low-to-moderate cut-off values. Therefore, we can conclude that the results would probably improve if studying a sample of the general population.
In conclusion, our study indicated that, with some limitations, the MicroDigitrapper-S portable device has a good sensitivity and specificity when used for the diagnosis of patients with suspected obstructive sleep apnoea. However, the overall agreement of automatic respiratory analysis with polysomnographic data when considering severe patients, was low. Using the semiautomatic analysis, with visual inspection and correction of respiratory events, the sensitivity and the negative predictive value increased, and the existing high specificity and positive predictive value were not altered. Therefore, we recommend the use of the MicroDigitrapper-S for diagnosis of obstructive sleep apnoea at low cut-offs, but for estimation of severity we advise that semiautomatic visual scoring should be utilized to obtain acceptable results.

\section{References}

1. Ferini-Strambi L, Zucconi M, Palazzi S, et al. Snoring and nocturnal oxygen desaturations in an Italian middleaged male population: epidemiologic study with an ambulatory device. Chest 1994; 105: 1759-1764.

2. Young T, Plata M, Dempsey J, Skatrud J, Weber S, Badr $\mathrm{S}$. The occurrence of sleep-disordered breathing among middle-aged adults. N Engl J Med 1993; 328: 12301235.

3. Stiller RA, Strollo PJ, Sanders MH. Unattended recording in the diagnosis and treatment of sleep-disordered breathing: unproven accuracy, untested assumptions and unready for routine use. Chest 1994; 105: 1306-1309.

4. Ferber R, Millman R, Coppola M, et al. Portable recording in the assessment of obstructive sleep apnea. Sleep 1994; 17: 378-392.

5. Standard of Practice Committee of the American Sleep Disorders Association. Practice parameters for the use of portable recording in the assessment of obstructive sleep apnea. Sleep 1994; 17: 372-377.

6. Johns NW. Daytime sleepiness, snoring and obstructive sleep apnea: the Epworth Sleepiness Scale. Chest 1993; 103: $30-36$.

7. Rechtschaffen A, Kales A. A manual of standardized terminology, techniques and scoring system for sleep stages of human subjects. Los Angeles, University of California at Los Angeles, 1968.

8. Guilleminault C. Sleeping and Waking disorders: indications and techniques. Menlo Park, California, AddisonWensley, 1982.

9. Emsellem HA, Corson WA, Rappaport BA, Hackett S, Smith LG, Hausfeld JN. Verification of sleep apnea using a portable sleep apnea screening device. South Med J 1990; 83: 748-752.

10. Orr WC, Eiken T, Pegram V, Jones R, Rundell OH. A laboratory validation study of a portable system for remote 
recording of sleep-related respiratory disorders. Chest 1994; 105: 160-162.

11. Bland JM, Altman DG. Statistical methods for assessing agreement between two methods of clinical measurement. Lancet 1986; i: 307-310.

12. White DP, Gibb TJ, Wall JM, Westbrook PR. Assessment of accuracy and analysis time of a novel device to monitor sleep and breathing in the home. Sleep 1995; 18: 115-126.

13. Flenley DC. Sleep in chronic obstructive lung disease. Clin Chest Med 1985; 6: 51-61.

14. He J, Kryger MH, Zorick FJ, Conway W, Roth T. Mortality and apnea index in obstructive sleep apnea: experience in 385 male patients. Chest 1988; 94: 9-14.

15. Gyulay S, Gould D, Sawyier B, Pond D, Mant A, Saunders N. Evaluation of a microprocessor-based portable home monitoring system to measure breathing during sleep. Sleep 1987; 10: 130-142.

16. Ancoli-Israel S, Kripke DF, Mason W, Messin S. Comparisons of home sleep recording and polysomnography in older adults with sleep disorders. Sleep 1981; 4: 283-291.

17. Svanborg E, Larsson H, Carlsson-Nordlander B, Pirskanen R. A limited diagnostic investigation for sleep apnea syndrome. Oximetry and static charge sensitive bed. Chest 1990; 98: 1341-1345.

18. Redline S, Tosteson T, Boucher MA, Millman R. Measurements of sleep-related breathing disturbances in epidemiologic studies: assessment of the validity and reproducibility of a portable monitoring device. Chest 1991; 100: 1281-1286.

19. Stoohs R, Guilleminault C. MESAM 4: an ambulatory device for detection of patients at risk for obstructive sleep apnea syndrome (OSAS). Chest 1992; 101: 12211227

20. Issa G, Morrison D, Hadjiuk E, Iyer A, Feroah T, Remmers JE. Digital monitoring of sleep-disordered breathing using sound and arterial oxygen saturation. Am Rev Respir Dis 1993; 148: 1023-1029.

21. Gyulay S, Olson LG, Hensley MJ, King MT, Muree Allen K, Saunder NA. A comparison of clinical assessment and home oximetry in the diagnosis of obstructive sleep apnea. Am Rev Respir Dis 1993; 147: 50-53.

22. Koziej M, Cieslicki JK, Gorzelak K, Sliwinski P, Zielinki J. Hand scoring of MESAM 4 recordings is more accurate than automatic analysis in screening for obstructive sleep apnoea. Eur Respir J 1994; 7: 1771-1175. 